\title{
The Role of Apelin and Apelin Receptor Expression in Migration and Invasiveness of Colon Cancer Cells
}

\author{
MARTA PODGÓRSKA*, KATARZYNA PIETRASZEK-GREMPLEWICZ*, \\ JOANNA OLSZAŃSKA and DOROTA NOWAK
}

Department of Cell Pathology, Faculty of Biotechnology, University of Wrocław, Wroclaw, Poland

\begin{abstract}
Background/Aim: Colon cancer is the second deadliest malignancy for human. Its correlation with obesity has led to an increasing number of studies focusing on the role of adipokines in colon cancer development. Apelin, which belongs to the family of adipokines, affects several pathological processes, including heart diseases, obesity and carcinogenesis. In this study, we examined the importance of apelin and apelin receptor (APJ) during motility regulation of colon cancer cells. Materials and Methods: Colon cancer cells with overexpression of apelin receptor, as well as cells with down-regulation of apelin were used in this study. Migration and invasion ability was tested using Transwell ${ }^{\circledR}$ filters. The proteolytic activity was analyzed with fluorescentsubstrate degradation assay and gelatin zymography. We also used confocal microscopy to examine migratory protrusion formation and the localization of MT1-MMP. The levels of AKT and ERK kinases were evaluated using Western blotting assay. Results: Overexpression of APJ receptor resulted in increased migration and invasion abilities through stimulation of migratory protrusion formation and proteolytic activity. These processes were mediated by PI3K/AKT and $M A P K$ signaling pathways. Opposite effect was obtained when the level of apelin was down-regulated. Conclusion: The level of apelin and its receptor is strictly connected with regulation of migration and invasion of colon cancer cells. Therefore, apelinergic system seems to be a promising target for anti-cancer therapy.
\end{abstract}

This article is freely accessible online.

*These Authors contribute equally to this paper.

Correspondence to: Dorota Nowak, Department of Cell Pathology, Faculty of Biotechnology, University of Wrocław, Wrocław, Poland. Tel: +48 713756289, e-mail:dorota.nowak@uwr.edu.pl

Key Words: Obesity, adipokines, apelin, apelin receptor, colon cancer, APLN, APJ.
Colorectal cancer (CRC) is the second deadliest malignancy for all the human population. The lifetime risk of developing CRC is about 1 in 23 for men and 1 in 25 women, while it has been shown that it is strongly associated with environmental and lifestyle factors, such as diet, tobacco, alcohol, androgen deprivation therapy, and obesity (1). A meta-analysis showed that elevated body mass index (BMI) is positively correlated with CRC development. There are several evidences for this association including changes in insulin-like growth factor 1 signaling, sex hormones, systemic inflammation and altered expression of adipokines (2).

Adipose tissue not only regulates energy homeostasis, but is also a part of endocrine system secreting adipokines, growth factors, cytokines and chemokines. Adipokines are essential mediators of various metabolic processes, such as gluconeogenesis, glucose uptake, insulin signaling and fatty acid oxidation. During the development of obesity, the excess of adipose tissue results in increased adipokines secretion, that lead to protection against some of the adverse metabolic consequences of obesity (3). However, several studies have shown that increased level of adipokines, such as apelin, could be connected with higher probability to develop cancer, including gastroesophageal cell carcinoma (4), gastric cancer (5), non-small cell lung carcinoma (6), bladder cancer (7), prostate cancer (8), ovarian cancer (9), and breast cancer (10).

The apelin-encoding gene $(A P L N)$ is located on chromosome Xq25-26.1 and encodes the 77-amino acid preproapelin. This prepropeptide may generate several apelin fragments: a 36-amino acid peptide (apelin-36), a 17-amino acid peptide (apelin-17) and a 13-amino acid peptide (apelin13). The latter may undergo pyroglutamylation at its $\mathrm{N}$ terminal glutamine residue producing the [Pyr1]apelin-13 form, what protects it from exopeptidase degradation $(11,12)$. Apelin is a ligand for the $\mathrm{G}$ protein-coupled receptor APJ, encoded by the APLNR gene. Both apelin and APJ create the apelinergic system and are involved in many physiological processes such as regulation of cardiovascular function, angiogenesis, fluid homeostasis and energy metabolism (13). Under pathological conditions, apelin and APJ might be related with the regulation of tumor growth, cancer cell migration, and even metastasis 
induction. Various apelin fragments could stimulate migration and invasion of different types of cancer cells, such as human lung adenocarcinoma (14), gastric cancer (5), and oral squamous cell carcinoma (15) through the mitogen-activated protein kinase/extracellular signal regulated kinase (MAPK/ERK) and phosphatidyl inositol 3-kinase/protein kinase B (PI3K/AKT) signaling pathways (13).

Our previous study indicated the increased level of apelin and its receptor in histologically confirmed colorectal cancer samples in comparison to normal tissue. Moreover, we noticed a strong correlation between apelin serum level and stage of CRC progression (16). Furthermore, we demonstrated that exogenous apelin added to the culture medium increases the migration and invasion abilities of colon cancer cells. Several apelin peptides were used to show that this adipokine stimulates the migratory protrusion formation, as well as proteolytic activity of the examined cells (17). Moreover, several other studies have examined the effect of apelin on cancer cell biology, by adding recombinant apelin to the culture medium (14, 18-21), omitting the fact that cancer cells also express and secrete this adipokine. Therefore, the aim of this study was to analyze whether overexpression of APLNR and silencing of $A P L N$ gene affect colon cancer cell migration .

\section{Materials and Methods}

Chemicals and reagents. Antibodies against ezrin were from Sigma (St. Louis, MO, USA), pAKT, AKT and GAPDH from Santa Cruz Biotechnology (Santa Cruz Biotechnology Inc., Santa Cruz, CA, USA), pERK, ERK and horseradish peroxidase (HRP)-conjugated secondary antibodies from Cell Signaling (Danvers, MA, USA), MT1-MMP from Merck Millipore (Darmstadt, Germany). The secondary antibodies Alexa Fluor $^{\circledR} 488$ goat anti-rabbit immunoglobulin G (IgG), Phalloidin Alexa Fluor ${ }^{\circledR} 568$ and Hoechst 33342 were from Invitrogen (Carlsbad, CA, USA). Matrigel ${ }^{\mathrm{TM}}$ was from Corning ${ }^{\circledR}$ (New York, NY, USA).

Cell culture. The human colorectal carcinoma cell line LS180 was obtained from Deutsche Krebsforschungzentrum, Heidelberg, Germany. The cell line was confirmed by LGC Cell Line Authentication Service. Cells were grown in MEM- $\alpha$ medium (Corning ${ }^{\circledR}$, New York, NY, USA) containing 10\% fetal bovine serum (FBS), $2 \mathrm{mM}$ glutamine and antibiotics $(100 \mathrm{U} / \mathrm{ml}$ penicillin, $100 \mu \mathrm{g} / \mathrm{ml}$ streptomycin) (Sigma, St. Louis, MO, USA). Cells were cultured in tissue culture flasks (Sarstedt Inc., Sarstedt, Germany) at $37^{\circ} \mathrm{C}$ in a $5 \% \mathrm{CO}_{2}, 95 \%$ humidified atmosphere and passaged twice a week using $0.25 \%$ trypsin/0.05\% EDTA solution (IITD PAN, Wrocław, Poland).

Transfection procedure. Cells were transfected with pCMV3APLNR-Flag plasmid (Sino Biological Inc., Beijing, PR China) or empty pCMV plasmid (MOCK) constituted control cells. To silence the APLN gene, cells were transfected with 29-mer small hairpin RNA (shRNA) constructs directed against human apelin or 29-mer non-targeting shRNA (shCTRL), which were purchased from OriGene (Rockville, MD, USA). Lipofectamine 3000 (Invitrogen,
Carlsbad, CA, USA) was used to transfect the cells according to the manufacturer's protocol. Transfected cells were selected for 2 weeks using antibiotics: hygromycin $(1 \mathrm{mg} / \mathrm{ml})$ or puromycin $(1 \mu \mathrm{g} / \mathrm{ml})$ (Santa Cruz Biotechnologies, Santa Cruz, CA, USA) for pCMV or shRNA constructs, respectively. Expression of APJ and apelin in all obtained cells was monitored by real-time PCR and ELISA methods.

qRT-PCR analysis of gene expression. To measure the expression level of APJ and apelin in the obtained cell lines, total RNA was isolated using the Universal RNA Purification Kit (EurX, Gdańsk, Poland), following the manufacturer's protocol. After DNase I (EurX, Gdańsk, Poland) treatment, reverse transcription reaction was performed with $0.5 \mu \mathrm{g}$ of RNA and the High Capacity cDNA Reverse Transcription Kit (Applied Biosystems, Foster City, CA, USA) following the manufacturer's instructions. Apelin and apelin receptor mRNA expression levels were determined with a real-time PCR reaction using StepOne Plus Real-Time PCR (Applied Biosystems, Foster City, CA, USA). The qPCR reaction mixture contained $3 \mu \mathrm{l}$ cDNA, $5 \mu \mathrm{l} \mathrm{TaqMan}{ }^{\mathrm{TM}}$ Fast Universal PCR Master Mix (Thermo Fisher Scientific, Waltham, MA, USA), $0.5 \mu 1$ TaqMan $^{\mathrm{TM}}$ MGB Probe [glyceraldehyde 3 phosphate dehydrogenase (GAPDH): Hs02758991_g1, APLN: Hs00936329_m1, APLNR: Hs00270873_s1] (Thermo Fisher Scientific, Waltham, MA, USA) and $1.5 \mu \mathrm{l}$ water. Relative quantification of gene expression was calculated based on the comparative CT (threshold cycle value) method ( $\triangle \mathrm{CT}=\mathrm{CT}$ gene of interest $-\mathrm{CT}$ housekeeping gene) (22). Three independent experiments were performed for all cell lines.

Migration and invasion assay. Cell migration and invasion assays were performed with Transwell ${ }^{\mathrm{TM}}$ filters (BD Biosciences, Franklin Lakes, NJ, USA) placed in 24-well plates. Before the experiment, cells were starved in serum-free MEM- $\alpha$ medium for $6 \mathrm{~h}$. Next, cells were seeded $\left(5 \times 10^{4}\right.$ cells/well $)$ in serum-free MEM- $\alpha$ directly onto rehydrated Transwell ${ }^{\mathrm{TM}}$ insert for migration or coated with Matrigel ${ }^{\mathrm{TM}}$ $(0.5 \mathrm{mg} / \mathrm{ml}$ in serum-free MEM- $\alpha$ medium) for invasion assay. The lower part of the well was filled with MEM- $\alpha$ medium containing $20 \%$ FBS and $5 \mathrm{nM}$ EGF was used as a chemoattractant for migration or invasion assay, respectively. After $24 \mathrm{~h}$, cells that migrated through the membrane were fixed with $4 \%$ formaldehyde, stained with Hoechst 33342 and counted using fluorescent microscope. The results are showed as a relative migration or invasion factor (\% of control). The experiments were performed three times and each independent experiment consisted of three replicates.

Western blotting assay. Twenty-four hours after cell seeding onto 6well plates, cells were lysed on ice with RIPA lysis buffer $(50 \mathrm{mM}$ Tris- $\mathrm{HCl}, \mathrm{pH} 7,4,150 \mathrm{mM} \mathrm{NaCl}, 1 \%$ Triton X-100, $1 \%$ sodium deoxycholate, $0,1 \%$ SDS, $1 \mathrm{mM}$ EDTA) or urea buffer $(50 \mathrm{mmol} / 1$ TRIS-HCl pH 7.4, 5\% SDS, $8.6 \%$ sucrose, 1 mmol/L DTT, $0.45 \%$ urea) supplemented with protease and phosphatase inhibitors cocktails (Sigma, St. Louis, MO, USA) for ezrin or kinase level determination, respectively. After three frozen-thawed cycles and centrifugation in $12,000 \times \mathrm{g}, 10 \mathrm{~min}, 4^{\circ} \mathrm{C}$, supernatants were collected, and the protein concentration was determined by Bradford procedure (23). Identical protein quantity was separated by sodium dodecyl sulphate-polyacrylamide gel (10\% acrylamide/bis $)$ electrophoresis (SDS-PAGE) and transferred onto nitrocellulose membranes (GE Healthcare, Chicago, IL, USA). After transfer, total protein was estimated by Ponceau S staining. Target proteins were probed with specific antibodies and detected using Western blotting 
Luminol Reagent (Bio-Rad, Hercules, CA, USA) under ChemiDoc (Bio-Rad, Hercules, CA, USA) and analyzed with ImageLab software (ver. 6.0, Bio-Rad, Hercules, CA, USA). The experiments were performed three times.

APJ and apelin protein level determination. The concentrations of human apelin-36 and human apelin receptor in the cell lysates were measured with commercially available enzyme-linked immunosorbent assay (ELISA) kits (apelin-36: MBS2021970, apelin receptor: MBS089535, MyBioSource Inc., San Diego, CA, USA) according to the manufacturer's instructions. The sensitivity of the apelin assay was $2.63 \mathrm{pg} / \mathrm{ml}$ and the apelin receptor assay was $1.0 \mathrm{ng} / \mathrm{ml}$.

Fluorescent staining. Twenty-four hours after cell seeding onto sterile coverslips in 24-well plates, cells were fixed with $4 \%$ formaldehyde and permeabilized with $0.1 \%$ Triton X-100. Then, coverslips were blocked with $1 \%$ bovine serum albumin and incubated with specific primary antibodies overnight in $4^{\circ} \mathrm{C}$. Next, the cells were washed three times in PBS and incubated with Phalloidin Alexa Fluor ${ }^{\circledR}$ 568, Hoechst 33342 and secondary antibodies conjugated with Alexa Fluor ${ }^{\circledR} 488$ for $1 \mathrm{~h}$ in room temperature. After washing with PBS, coverslips were mounted in Dako ${ }^{\circledR}$ cytomatic fluorescent mounting medium (Agilent Technologies, Santa Klara, CA, USA). Stained cells were visualized using confocal laser scanning microscope, Leica SP8, with LasX 3.3.0 software (Leica, Wetzlar, Germany). Images were processed using ImageJ software. Blebs quantification was calculated as a percent of cells forming blebs for 100 cells and recalculated as a percent of control. The experiments were performed three times. The number of MT1-MMPs positive signals was calculated for 100 cells using ImageJ software and quantitative analysis of focal adhesion protocol and presented as a percent of control.

Gelatin zymography. Gelatin zymography was performed to determine the activity of secreted metalloproteases in conditioned media. The media were collected after starvation of the cells for 48 $\mathrm{h}$ in serum-free MEM- $\alpha$ medium. Then, the media were concentrated with Amicon ${ }^{\circledR}$ Ultra-4 centrifugal filters (Merck Millipore, Darmstadt, Germany) and stored in $-20^{\circ} \mathrm{C}$. Samples were separated in SDS-polyacrylamide gels containing $1 \mathrm{mg} / \mathrm{ml}$ gelatin. Obtained gels were stained with Coomassie Brilliant Blue G-250 (Sigma, St. Louis, MO, USA), and metalloproteases (MMPs) activity was detected as transparent bands present on the blue background. The activity of MMPs was detected using ChemiDoc (Bio-Rad, Hercules, CA, USA). The experiments were performed three times.

Fluorescent-substrate degradation assay. The experiment was carried out using previously described protocol (24). Poly-L-lysine coated coverslips (BD Biosciences, Franklin Lakes, NJ, USA) were washed with PBS and fixed with $0.5 \%$ glutaraldehyde for $15 \mathrm{~min}$. Next, coverslips were coated with FITC-conjugated gelatin (Invitrogen, Carlsbad, CA, USA) for $10 \mathrm{~min}$. After wash with PBS, coverslips were incubated with sodium borohydride for $1 \mathrm{~min}$ and washed again with PBS. To determine the proteolytic activity, cells were seeded onto prepared coverslips $\left(5 \times 10^{4}\right.$ cells) and grown for $24 \mathrm{~h}$. Then, cells were fixed with $4 \%$ formaldehyde and stained with Phalloidin Alexa Fluor ${ }^{\circledR} 568$ for filamentous actin detection. Images were collected using confocal laser scanning microscope, Leica SP8, with LasX 3.3.0 software. Images were analyzed through quantification of darker areas with lack of fluorescence, which reflects activity of ECM degrading proteases using ImageJ software (25). The results were calculated for 100 cells as a percent of cells digesting gelatin. The experiments were performed three times and data were presented as a percent of control.

Statistical analysis. All statistical analyses were performed using the Statistica v. 13.0 software (StatSoft Inc., Tulsa, OK, USA). Data are presented as mean \pm standard deviation (SD) of three independent experiments. Statistical significance was determined using Student's $t$-test; the threshold for significance was set at $p \leq 0.05$.

\section{Results}

Characterization of generated cell lines. In our previous study we presented the influence of exogenous apelin peptides on invasiveness of the colon cancer cell lines LS180, EB3, 3LNLN and 5W. Various fragments of apelin were added to the culture medium and increased migration and invasion abilities of tested cells, through stimulation of migratory protrusion formation and proteolytic facilities activation (17). Therefore, in this study we aimed to examine the influence of endogenous apelinergic system on invasion of colon cancer cells. We used LS180 cells to produce cell line with up-regulated apelin receptor and a cell line with down-regulated apelin expression, since we observed that LS180 cells have low APJ and high apelin expression levels. The generated cells with up-regulated APLNR expression (APJ) and control cells transfected with empty plasmid (MOCK) were analyzed at mRNA and protein level, which confirmed overexpression of APLNR in the transfected cell lines (Figure 1A,C). Cell line with lowered expression of APLN gene (shAPLN) was produced using shRNA-based method. Stable down-regulation of apelin expression in the obtained cells in comparison with cells transfected with nontargeting shRNA (shCTRL) was validated at mRNA and protein level (Figure 1B, D).

Regulation of apelin and APJ expression affected migratory abilities of colon cancer cells. Several studies have suggested that apelin can be involved in the regulation of cancer cell migration. Therefore, we tested the migratory abilities of the transfected colon cancer cells. In LS180 cells, up-regulation of apelin receptor expression resulted in increased migration ability in comparison to MOCK cells (1.67-fold increase, $p=0.0035$ ), while the opposite effect was observed in the case of shAPLN cells with down-regulated apelin level (2-fold decrease, $p=0.0003$ ) (Figure 2A). The same results were obtained during assesment of invasion (for APJ cells: 2.05 -fold increase, $p=0.0004$; for shAPLN cells: 2.57 -fold decrease, $p=0.0006$ ) (Figure 2B). Migration ability is usually associated with the formation of migratory protrusions. LS180 cells are characterized by rounded morphology and facility to form spherical "blebs" protrusions (26). To verify whether APJ and apelin expression levels could also affect the formation of 
A

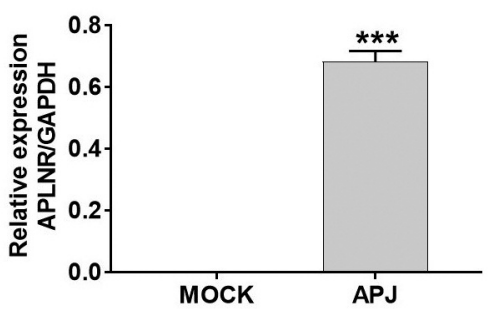

C

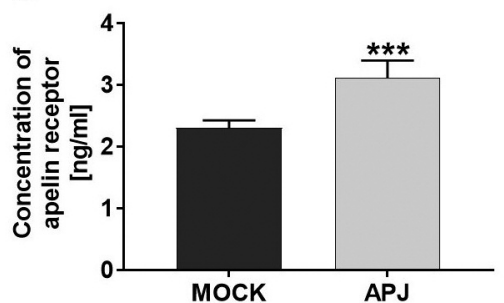

B

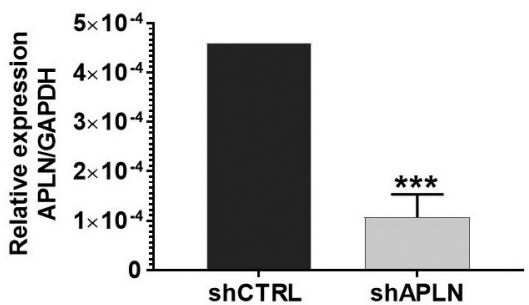

D

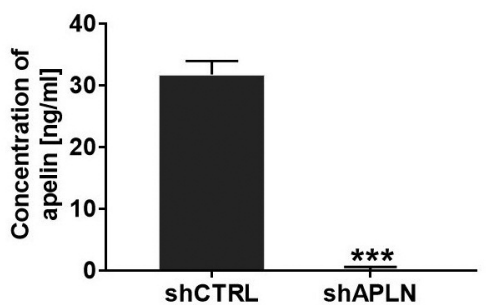

Figure 1. Expression level of apelin (APLN) and apelin receptor (APJ) in the generated variants of LS180 colon cancer cells. Result of qRT-PCR analysis of (A) APLNR or (B) APLN gene expression is shown as the mean of three independent experiments [relative expression compared to glyceraldehyde 3 phosphate dehydrogenase $(G A P D H) \pm S D)]$. ELISA results of $(C)$ APJ or (D) apelin protein level in generated cell lines. Protein level expressed ad the mean of three independent experiments. $p \leq 0.001$ (***).

migratory protrusions, the marker of blebs, ezrin, and filamentous actin, which is responsible for a shape of the cell, were both examined by immunocytochemistry (Figure $2 \mathrm{C})$. Fluorescence staining showed increased number of cells forming blebs in cells with overexpression of APJ (4-fold increase, $p=0.0008$ ) and decreased number in shAPLN cells (1.63-fold decrease, $p=0.0271$ ), which was confirmed by quantification of the number of cells forming blebs (Figure 2D). Additionally, the level of ezrin was determined using Western blotting method. We observed augmented level of blebs marker in APJ cells (2.38-fold increase, $p=0.0002)$, whereas no significant difference was observed in shAPLN cells (Figure 2E).

The effect of apelin and its receptor on the proteolytic abilities of colon cancer cells. Invasiveness of cancer cells, as well as the capability to metastasize, is connected with their ability to digest the elements of ECM (26). Therefore, the proteolytic activity of the generated cell lines was determined using gelatin-FITC degradation assay (Figure $3 \mathrm{~A})$. The obtained data showed increased ability to digest the gelatin in cells with up-regulated expression of apelin receptor (3.26-fold increase, $p=0.0042$ ). Down-regulation of apelin resulted in decreased proteases activity (3.23-fold decrease, $p=0.0006)$. Quantification of cells digesting gelatin confirmed previous observations (Figure 3B). Moreover, we performed gelatin zymography using concentrated conditioned media, which is another method to analyze the proteolytic activity of the cells (Figure 3C). Overexpression of APJ resulted in increased activity of MMP-2 (1.37-fold increase, $p=0.0034)$ and MMP-9 (1.28-fold increase, $p=0.0262)$. In case of shAPLN cells, the level of MMP-2 was decreased (2.86-fold decrease, $p=0.0002)$. Densitometric measurements confirmed our observation, while in shAPLN cells, the level of MMP-9 was also decreased compared to the control (2.99-fold decrease, $p=0.0003$ ) (Figure 3D). After analysis of secreted metalloproteases, we intended to examine the influence of apelin and its receptor on the level of membrane-type metalloprotease (MT1-MMP), different enzyme important for cancer invasiveness, using immunocytochemistry (Figure 4A). The level of MT1-MMP in APJ cells was higher than in MOCK cells (5.78-fold increase, $p=0.0001$ ), whereas in shAPLN cells the expression of this metalloprotease was decreased (2.17-fold decrease, $p=0.0001$ ), which was confirmed by quantification of the number of MT1-MMP positive signals (Figure 4B).

Influence of apelin and APJ expression on activation of signaling pathways. To verify that apelin and APJ stimulate migration and invasion of colon cancer cells through MAPK/ERK and PI3K/AKT signaling pathways, we tested the levels of phosphorylated forms of AKT and ERK kinases. In cells with up-regulated expression of apelin receptor the levels of active, phosphorylated forms of AKT and ERK kinases were augmented (Figure 5A), whereas the ratios of phosphorylated to total kinases level were similar in control and altered cells (Figure 5B). In cells with down-regulated level of apelin we did not observe any significant change in 
A

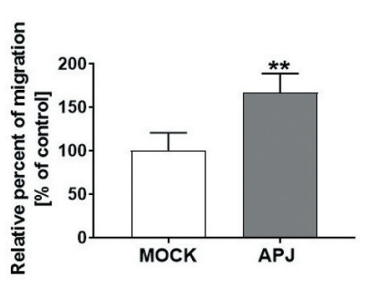

B

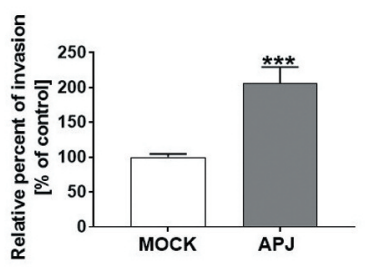

D

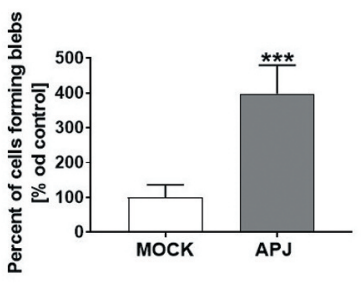

E

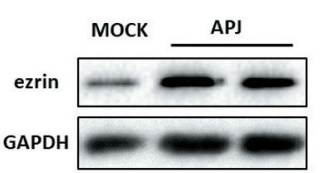

F

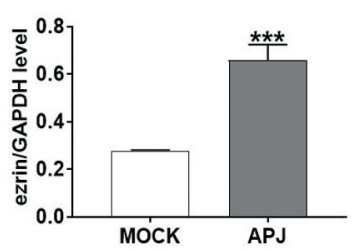

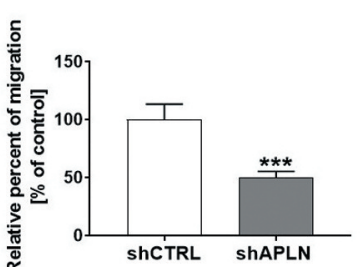
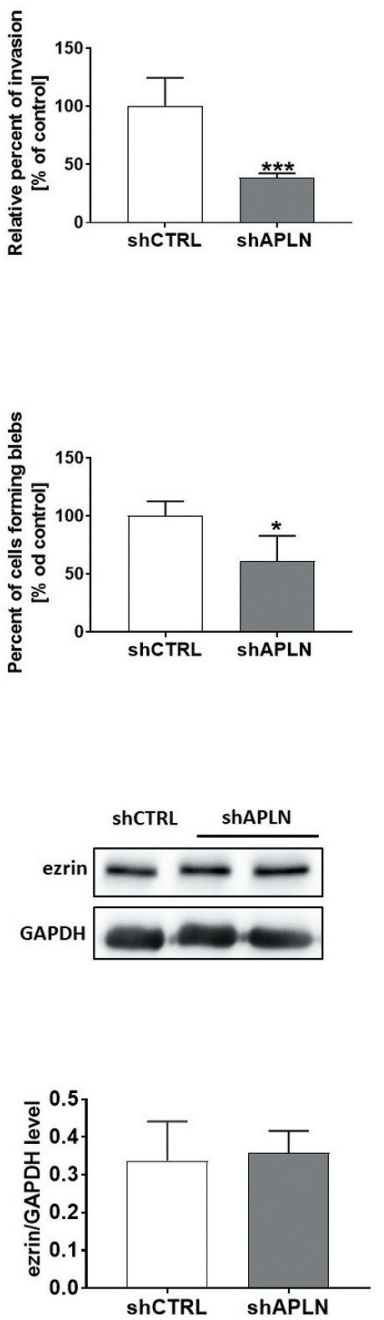

C
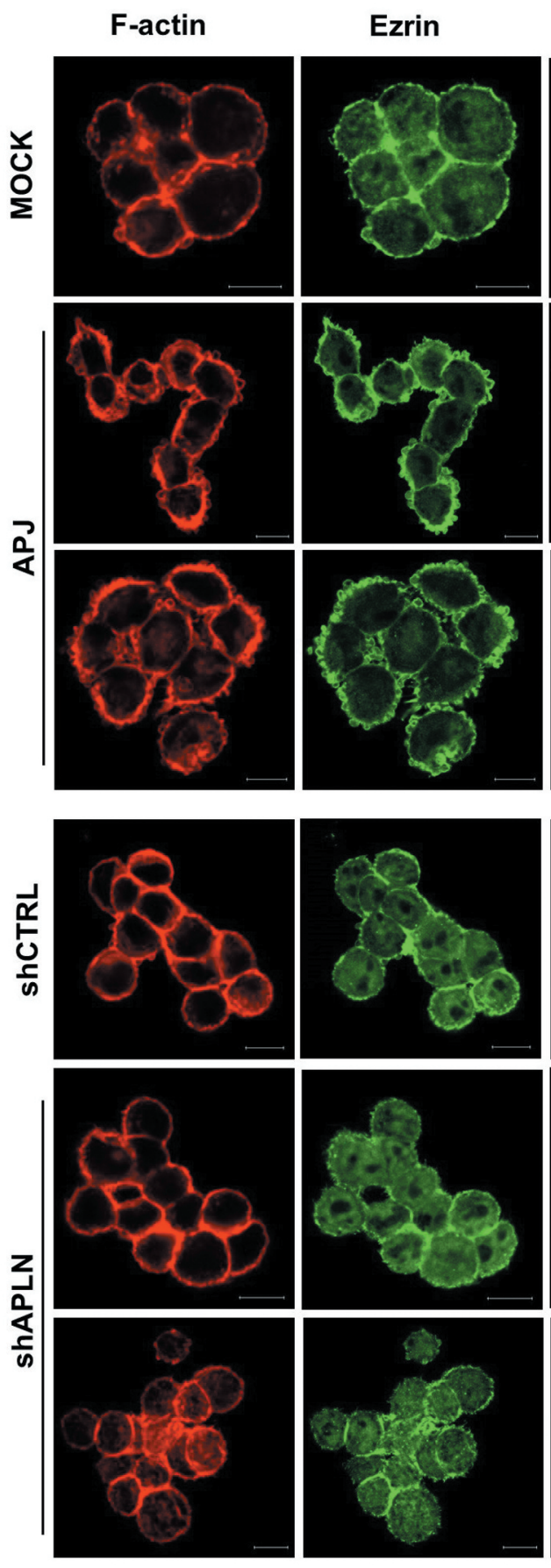

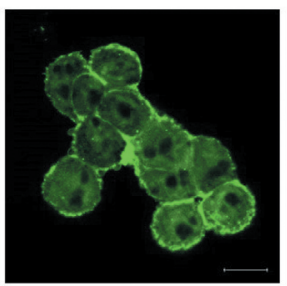

Ezrin
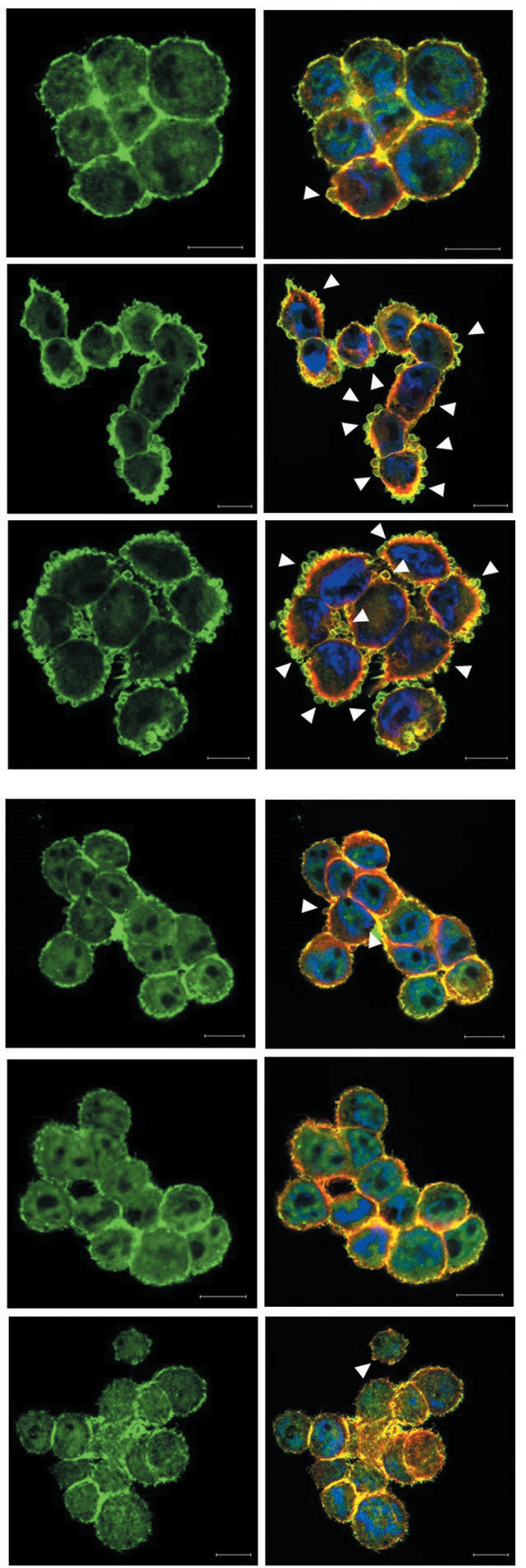

Figure 2. Migration and invasion abilities of colon cancer cells with altered level of apelin and apelin receptor (APJ). (A) Migration of cells seeded onto Transwell filters for $24 \mathrm{~h}$. (B) Invasion of cells seeded onto the layer of Matrigel in Transwell filters for 24 h. Relative migration/invasion factor was calculated versus control cells, where number of migrating control cells is set as $100 \%$. Results are expressed as the mean $\pm S D$ of three independent experiments. (C) Representative localization of ezrin (green), filamentous actin (red) and nuclei (blue) in generated cell lines. Arrowheads indicate migratory protrusions. Scale bars - $10 \mu \mathrm{m}$. (D) Quantification of the number of cells forming blebs shown as a percent of control cells, where number of control cells forming blebs is set as $100 \%$. Results are expressed as the mean \pm SD of three independent experiments. (E) Representative Western blot and $(F)$ densitometric analysis for level of ezrin in tested cells. The glyceraldehyde 3 phosphate dehydrogenase (GAPDH) level was shown as a loading control. Results are expressed as the mean \pm SD of three independent experiments. $p \leq 0.05(*), p \leq 0.01(* *), p \leq 0.001(* * *)$. 
A
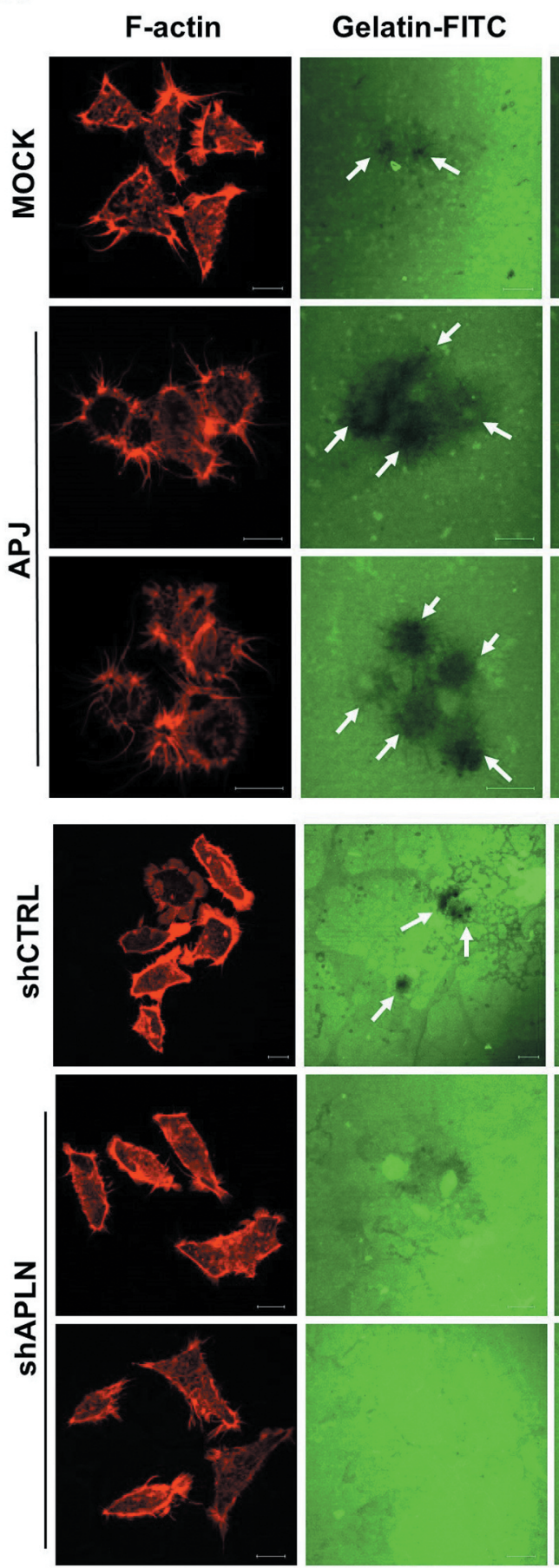

Merge
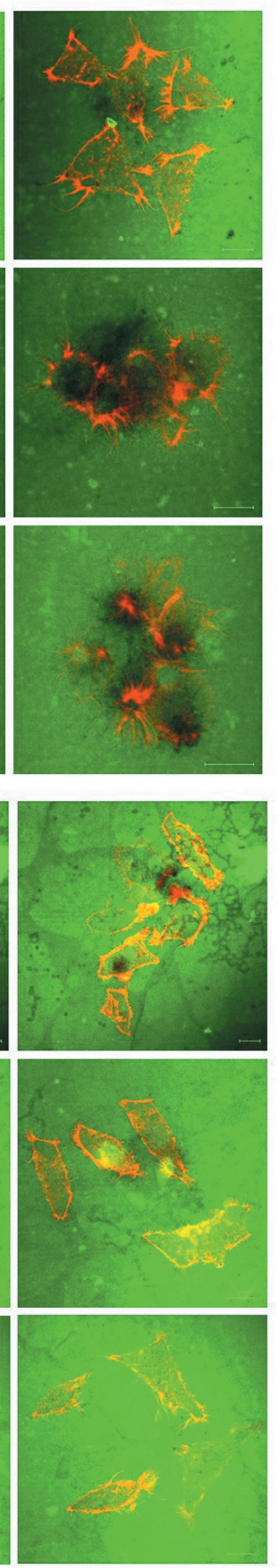

B

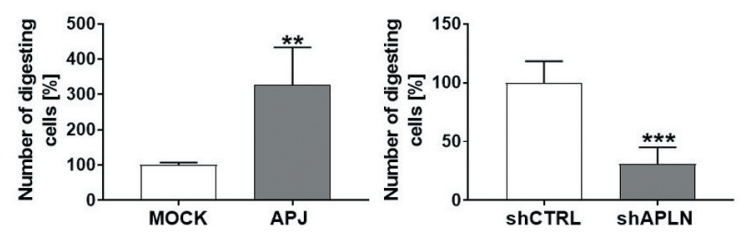

C

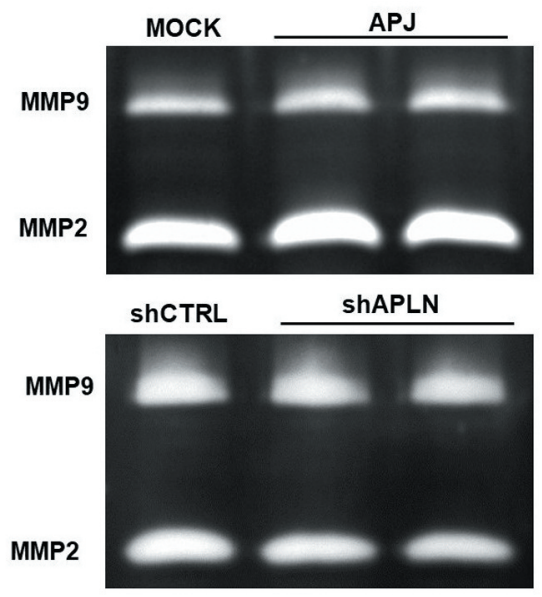

D
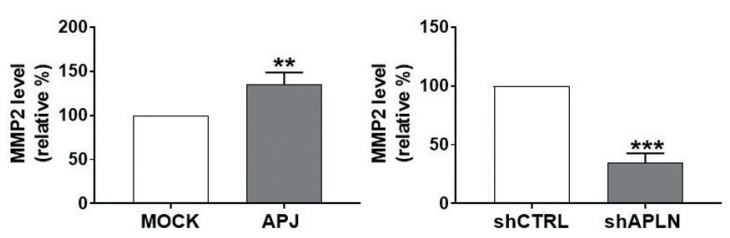

$\mathbf{E}$
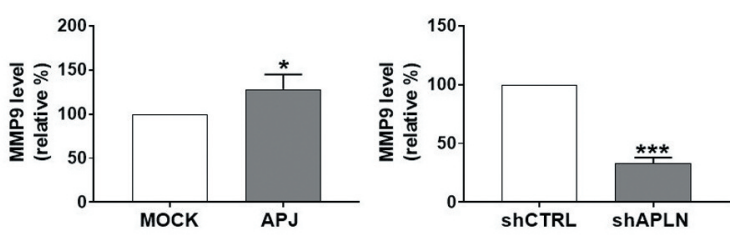

Figure 3. Proteolytic activity of colon cancer cells with modified apelin receptor (APJ) and apelin expression level. (A) The proteolytic activity of cells (shape of the cell - filamentous actin - red) using FITC-conjugated gelatin (green). Gelatin degradation areas visualized as the dark spots are indicated with white arrows. Scale bars - $10 \mu \mathrm{m}$. (B) Quantification of digestion area calculated using ImageJ software from at least 100 cells from three independent experiments. The number of control cells digesting gelatin is set as 100\%. (C) Metalloprotease-2 (MMP-2) and -9 (MMP-9) activity in concentrated conditioned media tested by gelatin zymography with densitometric analysis of MMP-2 (D) and MMP-9 level (E). Results are expressed as the mean $\pm S D$ of three independent experiments. $p \leq 0.05(*), p \leq 0.01(* *), p \leq 0.001(* * *)$. 
A MOCK

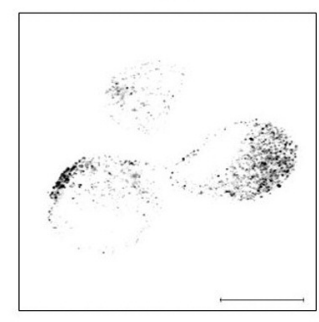

ShCTRL

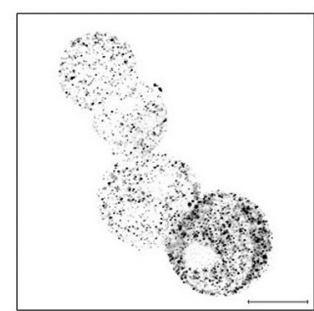

APJ

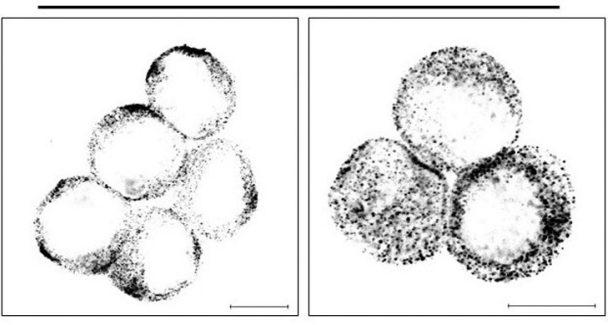

ShAPLN

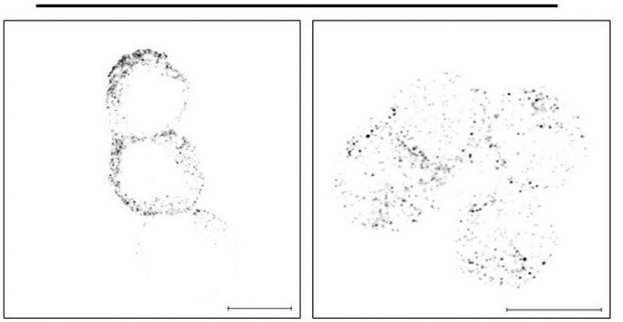

B
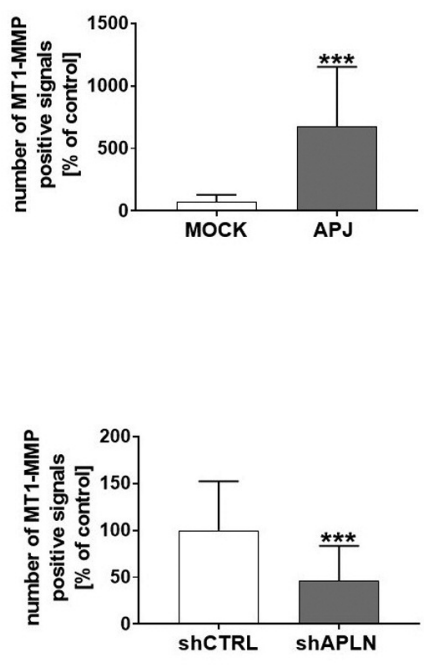

Figure 4. Impact of apelin and apelin receptor (APJ) expression on membrane type I-matrix metalloproteinase (MT1-MMP) level in LS180 cells. (A) The localization of MT1-MMP (black spots) in tested cells. Scale bars - $10 \mu \mathrm{m}$. (B) Quantification of the number of MT1-MMP positive signals. Results are expressed as the mean $\pm S D$ of three independent experiments. $p \leq 0.05(*), p \leq 0.01$ (**), $p \leq 0.001$ (***).

the levels of AKT and ERK kinases (Figure 5C, D).

\section{Discussion}

Overweight and obesity are defined by a World Health Organization (WHO) as an abnormal or excessive fat accumulation that present a risk to health. Nowadays, nearly a third of human population is classified as overweight or obese (27). Obesity is correlated with risk of several morbidities, such as type 2 diabetes, hypertension, stroke, coronary artery disease, asthma, osteoarthritis, pulmonary embolism, and cancer (28). Hormones released by adipocytes, called adipokines, play a key role in several physiological processes, including energy balance, appetite regulation, insulin sensitization, inflammatory response and vascular homeostasis (29).

Apelin is a secreted peptide that belongs to the family of adipokines (11). Several studies have demonstrated a potential link of apelin with obesity. In obese women the level of serum apelin was significantly increased in comparison to healthy controls and was positively correlated with abnormal metabolic parameters (30). The same result was observed in children, where serum level of apelin was higher in obese patients than in the control group (31). The correlation between obesity and cancer development is quite clear in human populations and being overweight increases the probability to develop cancer 1.5 to 2.4 -fold (32). In obese men with colon cancer, the level of plasma apelin was significantly increased compared to healthy individuals (33). Moreover, tumor-associated apelin-36 was positively correlated with the concentration of apelin receptor, while both expression levels were higher in colon cancer in comparison to healthy tissue (16). Additionally, this adipokine can stimulate tumor growth and proliferation of several types of cancer, including non-small cell lung cancer (34), prostate cancer (35), ovarian cancer (9), and cholangiocarcinoma (20). Numerous studies have indicated that the apelinergic system can be involved in migration and invasion of cancer cells, however the mechanism of its precise action is still unclear (13).

Migration and invasion of cancer cells are crucial processes during cancer development and spreading. Apelin has been shown to stimulate migration of gastric cancer cells (5), oral squamous cell carcinoma cells (36), and human lung adenocarcinoma cells (14). Moreover, apelin-13 has been demostrated to induce lymph node metastasis of implanted apelin-overexpressing melanoma cells in mice (37). Herein, migration assay revealed that up-regulation of APJ expression increased the ability of colon cancer cells to migrate, whereas cells with down-regulated apelin migrated poorly in comparison to control cells. The same results were obtained in a previous study on ovarian cancer cells, where overexpression of APJ led to increased migration of cells (38). In that study, up-regulation of apelin receptor was also associated with invasion and metastasis of ovarian cancer in vivo (38). Additionally, high expression of APLNR in hepatocellular carcinoma has been shown to be related with 
A

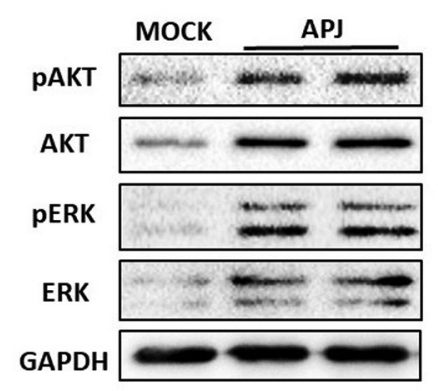

C
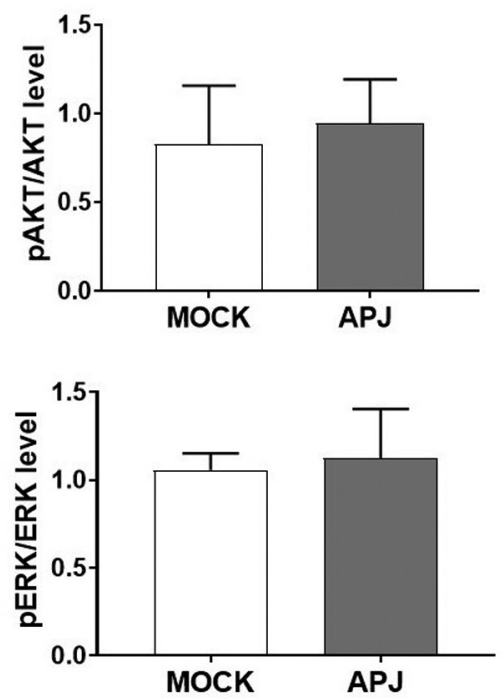

B

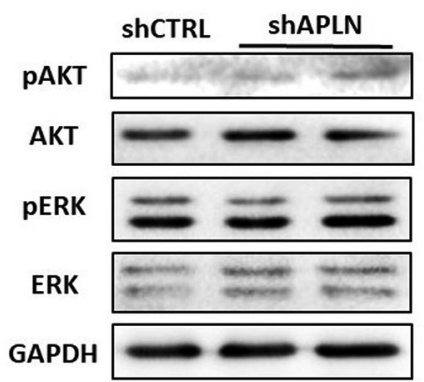

D
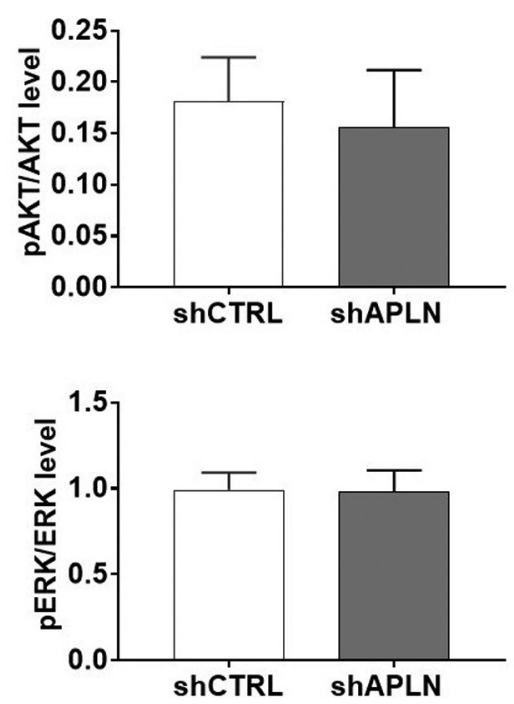

Figure 5. Effect of altered expression of apelin and apelin receptor (APJ) on activity of protein kinase B (AKT) and extracellular signal regulated kinase (ERK). Representative Western blot and densitometric analysis of kinases level in cells with overexpression of apelin receptor (A, C) or down-regulation of apelin $(B, D)$. Detection of glyceraldehyde 3 phosphate dehydrogenase (GAPDH) serves as a loading control. Results are expressed as the mean $\pm S D$ of three independent experiments.

microvascular invasion and intrahepatic metastasis (39). Moreover, apelin down-regulation has been shown to reduce metastasis of breast and lung cancer (40). Therefore, we investigated the invasion abilities of cancer cells with altered expression levels of APJ and apelin. Cells overexpressing APJ were characterized by increased ability to invade compared to the control cells. The opposite effect was observed in knocked-down APLN cells, which had decreased invasion facilities.

Migration of the cells is often connected with migratory protrusion formation. Cells characterized by rounded morphology are usually able to form specific protrusions called "blebs". These actin-rich structures can be formed relatively fast, suggesting that this protrusion type displays a capacity to explore the extracellular environment (41). Microscopic observations and quantification of cells forming migratory structures showed that down-regulation of apelin led to decreased ability to form blebs in comparison to control cells, in contrast to cells overexpressing APJ. Similar results were obtained in colon cancer cells. The number of cells forming blebs was increased, when the cells were stimulated with different apelin peptides, suggesting that apelinergic system is important in this process (17). We also determined the level of ezrin, which belongs to the family of actin-binding proteins and is essential during blebs formation. Together with other anchored proteins like radixin and moesin, ezrin interacts with plasma membrane leading to detachment of the membrane from actin filaments and formation of spherical membrane protrusion by intracellular pressure (42). Expression of bleb marker was augmented in APJ cells, however we did not observe any significant change in the level of ezrin in cells with down-regulated apelin. All these 
findings indicate that apelin/APJ system is important in regulation of migration and invasion of colon cancer cells.

Invasion of cancer cells is correlated with proteolytic activity. The ability of cells to digest ECM and invade through it is crucial process during metastasis development (43). Overexpression of APJ in colon cancer cells led to increased ability to digest gelatin in comparison to MOCK cells. On the other hand, down-regulation of apelin expression resulted in decreased activity of proteolytic enzymes, which was confirmed quantitatively. Since apelin is able to activate MMP-9 in gastric cancer (5), we performed gelatin zymography to test the level of MMP-9 and MMP-2. These enzymes are secreted gelatinases with the ability to cleave many different substrates, including ECM elements, cytokines or growth factors, that in turn regulate signaling pathways associated with migration and invasion processes (44). The activity of these proteases was decreased in shAPLN cells and was higher in APJ cells, compared to the control cells. Another metalloprotease crucial for cellular invasion is MT1-MMP which is overexpressed in many tumors (45). The expression of MT1-MMP is connected with poor prognosis in patients with advanced neuroblastoma, small cell lung cancer, head and neck carcinoma, bladder cancer, breast cancer, and ovarian cancer (45). The number of MT1-MMPpositive signals was increased in cells overexpressing APJ, whereas down-regulation of apelin lead to decreased MT1MMP level. All these data suggest that the expression levels of apelin and APJ expression level is important in proteolytic activity regulation of colon cancer cells.

The obtained results indicate that apelin and its receptor are both implicated in the regulation of migration and invasion processes by modulation of proteolytic abilities of colon cancer cells. Therefore, we tested whether altered expression of apelinergic system influences the activation of MAPK/ERK and PI3K/AKT signaling pathways, which are connected with the regulation of cell movement $(46,47)$. In cells with overexpression of APJ, the levels of both phosphorylated and total kinases AKT and ERK were augmented; however, the ratios of phosphorylated to total protein level of tested kinases were stable in both control and transfected cells, suggesting that overexpression of apelin receptor increases the expression level of AKT and ERK kinases, whereas the activation of signaling pathways is still the same. Our results are in line with previous studies, in which overexpression of APJ in ovarian cancer cells resulted in increased level of pAKT and pERK (38). Interestingly, the level of kinases was not altered in cells with down-regulated apelin expression. The level of ezrin was also stable in control and modified cells. It could be connected with the fact that apelin is not the only ligand that can activate the APJ receptor (48). Apela, which shares little sequence homology with apelin, has been recently identified as a second endogenous APJ receptor ligand (48). Therefore, the expression level of AKT and ERK kinases, as well as ezrin, could be the same after compensation of the loss of apelin with apela secretion. Moreover, apelin classified as a ligand could not have the same significant impact on signaling pathways as APJ classified as a G-protein-coupled receptor.

In conclusion, our study presents the effect of apelin and APJ protein level on the invasion ability of colon cancer cells. The obtained data suggest that not only secreted apelin, but also activation of endogenous apelinergic system is strictly connected with migration and invasion abilities of colon cancer cells, mostly because of the migratory protrusion formation and proteolytic activity regulation. Moreover, these processes are mediated via MAPK/ERK and PI3K/AKT signaling pathways. Therefore, apelin and its receptor present promising targets for anti-cancer therapy

\section{Conflicts of Interest}

The Authors confirm that there are no conflicts of interest.

\section{Authors' Contributions}

Conceptualization, D.N. and K.P.-G.; Methodology, M.P. and K.PG; Validation, M.P.; Formal Analysis, M.P. and K.P.-G.; Investigation, M.P., J.O. and K.P.-G.; Resources, D.N. Data Curation: M.P. and K.P.-G.; Writing - Original Draft Preparation, M.P., K.P.-G. and D.N.; Writing - Review \& Editing, M.P., K.P.-G., J.O. and D.N.; Visualization, M.P.; Supervision, D.N.; Project Administration, M.P.; Funding Acquisition, K.P.-G.

\section{Acknowledgements}

The Authors are grateful to Ilona Styczeń for technical support. This work was supported by the National Science Centre, Poland (project no. 2016/23/D/NZ5/00049, granted to K.P.G.).

\section{Data Statement}

The data that support the findings of this study are available from the corresponding author upon reasonable request.

\section{References}

1 Recio-Boiles A and Cagir B: Cancer, Colon, 117-117, 2018. Available at: https://www.ncbi.nlm.nih.gov/books/NBK470380 [Last accessed on December 9 2020]

2 Song M, Chan AT and Sun J: Influence of the gut microbiome, diet, and environment on risk of colorectal cancer. Gastroenterology 158: 322-340, 2020. PMID: 31586566. DOI: $10.1053 /$ j.gastro .2019 .06 .048

3 Chait A and den Hartigh LJ: Adipose tissue distribution , inflammation and its metabolic consequences, including diabetes and cardiovascular disease. Front Cardiovasc Med 7: 1-41, 2020. PMID: 32158768. DOI: 10.3389/fcvm.2020.00022

4 Diakowska D, Markocka-Mạczka K, Szelachowski P and Grabowski K: Serum levels of resistin, adiponectin, and apelin in gastroesophageal cancer patients. Dis Markers 2014: 619649, 2014. PMID: 25049439. DOI: 10.1155/2014/619649 
5 Feng M, Yao G, Yu H, Qing Y and Wang K: Tumor apelin, not serum apelin, is associated with the clinical features and prognosis of gastric cancer. BMC Cancer 16: 794, 2016. PMID: 27733135. DOI: 10.1186/s12885-016-2815-y

6 Berta J, Kenessey I, Dobos J, Tovari J, Klepetko W, Jan Ankersmit H, Hegedus B, Renyi-Vamos F, Varga J, Lorincz Z, Paku S, Ostoros G, Rozsas A, Timar J and Dome B: Apelin expression in human non-small cell lung cancer: role in angiogenesis and prognosis. J Thorac Oncol 5: 1120-1129, 2010. PMID: 20581707. DOI: 10.1097/JTO.0b013e3181e2c1ff

7 Yang L, Li Y-L, Li X-Q and Zhang Z: High apelin level indicates a poor prognostic factor in muscle-invasive bladder cancer. Dis Markers 2019: 4586405, 2019. PMID: 30984306. DOI: $10.1155 / 2019 / 4586405$

8 Wan Y, Zeng ZC, Xi M, Wan S, Hua W, Liu YL, Zhou YL, Luo HW, Jiang FN and Zhong W De: Dysregulated microRNA224/apelin axis associated with aggressive progression and poor prognosis in patients with prostate cancer. Hum Pathol 46: 295-303, 2015. PMID: 25532941. DOI: 10.1016/j.humpath.2014.10.027

9 Hoffmann M, Fiedor E and Ptak A: Bisphenol A and its derivatives tetrabromobisphenol $\mathrm{A}$ and tetrachlorobisphenol A induce apelin expression and secretion in ovarian cancer cells through a peroxisome proliferator-activated receptor gammadependent mechanism. Toxicol Lett 269: 15-22, 2017. PMID: 28111160. DOI: 10.1016/j.toxlet.2017.01.006

10 Salman T, Demir L, Varol U, Akyol M, Oflazoglu U, Yildiz Y, Taskaynatan H, Cengiz H, Guvendi G, Kucukzeybek Y, Alacacioglu A and Tarhan O: Serum apelin levels and body composition changes in breast cancer patients treated with an aromatase inhibitor. J BUON 21: 1419-1424, 2016. PMID: 28039702 .

11 Tatemoto K, Hosoya M, Habata Y, Fujii R, Kakegawa T, Zou MX, Kawamata Y, Fukusumi S, Hinuma S, Kitada C, Kurokawa $\mathrm{T}$, Onda $\mathrm{H}$ and Fujino M: Isolation and characterization of a novel endogenous peptide ligand for the human APJ receptor. Biochem Biophys Res Commun 251: 471-476, 1998. PMID: 9792798. DOI: 10.1006/bbrc.1998.9489

12 Masri B, Knibiehler B and Audigier Y: Apelin signalling: A promising pathway from cloning to pharmacology. Cell Signal 17: 415-426, 2005. PMID: 15601620. DOI: 10.1016/j.cellsig. 2004.09 .018

13 Wysocka MB, Pietraszek-Gremplewicz K and Nowak D: The role of apelin in cardiovascular diseases, obesity and cancer. Front Physiol 9: 1-15, 2018. PMID: 29875677. DOI: 10.3389/fphys.2018.00557

14 Lv D, Li L, Lu Q, Li Y, Xie F, Li H, Cao J, Liu M, Wu D, He L and Chen L: PAK1-cofilin phosphorylation mediates human lung adenocarcinoma cells migration induced by apelin-13. Clin Exp Pharmacol Physiol 43: 569-579, 2016. PMID: 26918678. DOI: 10.1111/1440-1681.12563

15 Hao Y, Li M, Ning F and Wang X: APJ is associated with treatment response in gastric cancer patients receiving concurrent chemoradiotherapy and endostar therapy. Cancer Biother Radiopharm 32: 133-138, 2017. PMID: 28514205. DOI: 10.1089/cbr.2016.2138

16 Podgórska, Diakowska, Pietraszek-Gremplewicz, Nienartowicz and Nowak: Evaluation of apelin and apelin receptor level in the primary tumor and serum of colorectal cancer patients. J Clin Med 8: 1513, 2019. PMID: 31547096. DOI: 10.3390/jcm 8101513
17 Podgórska M, Pietraszek-Gremplewicz K and Nowak D: Apelin effects migration and invasion abilities of colon cancer cells. Cells 7: 113, 2018. PMID: 30127323. DOI: 10.3390/cells7080113

18 Hashimoto Y, Ishida J, Yamamoto R, Fujiwara K, Asada S, Kasuya Y, Mochizuki N and Fukamizu A: G protein-coupled APJ receptor signaling induces focal adhesion formation and cell motility. Int J Mol Med 16: 787-792, 2005. PMID: 16211245 .

19 Picault FX, Chaves-Almagro C, Projetti F, Prats H, Masri B and Audigier Y: Tumour co-expression of apelin and its receptor is the basis of an autocrine loop involved in the growth of colon adenocarcinomas. Eur J Cancer 50: 663-674, 2014. PMID: 24316062. DOI: $10.1016 /$ j.ejca.2013.11.017

20 Hall C, Ehrlich L, Venter J, O'Brien A, White T, Zhou T, Dang T, Meng F, Invernizzi P, Bernuzzi F, Alpini G, Lairmore T and Glaser S: Inhibition of the apelin/apelin receptor axis decreases cholangiocarcinoma growth. Cancer Lett 386: 179-188, 2017. PMID: 27894959. DOI: 10.1016/j.canlet.2016.11.025

21 Chen T, Liu N, Xu G, Liu T, Liu Y and Zhou Y: Apelin13 / APJ promotes proliferation of colon carcinoma by activating Notch3 signaling pathway. Oncotarget 8: 101697-101706, 2017. PMID: 29254197. DOI: $10.18632 /$ oncotarget.21904

22 Livak KJ and Schmittgen TD: Analysis of relative gene expression data using real-time quantitative PCR and the 2$\Delta \Delta C T$ method. Methods 25: 402-408, 2001. PMID: 11846609 . DOI: $10.1006 /$ meth.2001.1262

23 Bradford MM: A rapid and sensitive method for the for the quantitation of microgram quantities of protein utilizing the principle of protein dye-binding. Anal Biochem 72: 248-254, 1976. PMID: 942051. DOI: 10.1016/0003-2697(76)90527-3

24 Artym V V., Zhang Y, Seillier-Moiseiwitsch F, Yamada KM and Mueller SC: Dynamic interactions of cortactin and membrane type 1 matrix metalloproteinase at invadopodia: Defining the stages of invadopodia formation and function. Cancer Res 66: 3034-3043, 2006. PMID: 16540652. DOI: 10.1158/00085472.CAN-05-2177

25 Gialeli C, Theocharis AD and Karamanos NK: Roles of matrix metalloproteinases in cancer progression and their pharmacological targeting. FEBS J 278: 16-27, 2011. PMID: 21087457. DOI: $10.1111 /$ j.1742-4658.2010.07919.x

26 Nowak D, Mazur AJ, Popow-Woźniak A, Radwańska A, Mannherz HG and Malicka-Błaszkiewicz M: Subcellular distribution and expression of cofilin and ezrin in human colon adenocarcinoma cell lines with different metastatic potential. Eur J Histochem 54: e14, 2010. PMID: 20558337. DOI: 10.4081/ejh.2010.e14

27 Chooi YC, Ding C and Magkos F: The epidemiology of obesity. Metabolism 92: 6-10, 2019. PMID: 30253139. DOI: 10.1016/ j.metabol.2018.09.005

28 Djalalinia S, Qorbani M, Peykari N and Kelishadi R: Health impacts of obesity. Pak J Med Sci 31: 239-242, 2015. PMID: 25878654. DOI: $10.12669 /$ pjms.311.7033

29 Gómez-Hernández A, Beneit N, Díaz-Castroverde S and Escribano Ó: Differential role of adipose tissues in obesity and related metabolic and vascular complications. Int J Endocrinol 2016: 1216783, 2016. PMID: 27766104. DOI: $10.1155 / 2016 / 1216783$

30 Zaki M, Kamal S, Ezzat W, Hassan N, Yousef W, Ryad H, Mohamed R, Youness E, Basha W and Elhosary Y: Serum apelin levels and metabolic risk markers in obese women. J Genet Eng Biotechnol 15: 423-429, 2017. PMID: 30647682. DOI: 10.1016/j.jgeb.2017.05.002 
31 El Wakeel MA, El-Kassas GM, Kamhawy AH, Galal EM, Nassar MS, Hammad EM and El-Zayat SR: Serum apelin and obesity-related complications in Egyptian children. Open Access Maced J Med Sci 17: 1354-1358, 2018. PMID: 30159056. DOI: 10.3889/oamjms.2018.312

32 Stone TW, McPherson M and Gail Darlington L: Obesity and cancer: Existing and new hypotheses for a causal connection. EBioMedicine 30: 14-28, 2018. PMID: 29526577. DOI: 10.1016/j.ebiom.2018.02.022

33 Al-Harithy RN and Al-Otaibi WA: Apelin-12 levels in obese patients with colon cancer. Cancer Immunol Immunother 1: 1$5,2015$.

34 Berta J, Kenessey I, Dobos J, Tovari J, Klepetko W, Jan Ankersmit H, Hegedus B, Renyi-Vamos F, Varga J, Lorincz Z, Paku S, Ostoros G, Rozsas A, Timar J and Dome B: Apelin expression in human non-small cell lung cancer: role in angiogenesis and prognosis. J Thorac Oncol 5: 1120-1129, 2010. PMID: 20581707. DOI: 10.1097/JTO.0b013e3181e2c1ff

35 Tekin S, Sandal S and Colak C: Effects of Apelin-13 on human prostate cancer lines [İnsan Prostat Kanseri Hücre Serilerinde Apelin-13'ün Etkileri]. Med Sci 303: 1427-418143, 2014. DOI: 10.5455/medscience.2014.03.8143

36 Heo K, Kim JH, Sung HJ, Li HJ, Yoo CW, Kim JY, Park JY, Lee UL, Nam BH, Kim EO, Kim SY, Lee SH, Park JB and Choi SW: Hypoxia-induced up-regulation of apelin is associated with a poor prognosis in oral squamous cell carcinoma patients. Oral Oncol 48: 500-506, 2012. PMID: 22285858. DOI: 10.1016/j.oraloncology.2011.12.015

37 Berta J, Hoda MA, Laszlo V, Rozsas A, Garay T, Torok S, Grusch M, Berger W, Paku S, Renyi-Vamos F, Masri B, Tovari J, Groger M, Klepetko W, Hegedus B and Dome B: Apelin promotes lymphangiogenesis and lymph node metastasis. Oncotarget 5: 4426-4437, 2014. PMID: 24962866. DOI: 10.18632/oncotarget.2032

38 Neelakantan D, Dogra S, Devapatla B, Jaiprasart P, Mukashyaka MC, Janknecht R, Dwivedi SKD, Bhattacharya R, Husain S, Ding $\mathrm{K}$ and Woo S: Multifunctional APJ pathway promotes ovarian cancer progression and metastasis. Mol Cancer Res 17: 1378-1390, 2019. PMID: 30858172. DOI: 10.1158/15417786.MCR-18-0989

39 Lee T, Park C-K and Ha SY: Prognostic role of apelin receptor expression in hepatocellular carcinoma treated with curative surgical resection. Anticancer Res 39: 3025-3031, 2019. PMID: 31177144. DOI: 10.21873 /anticanres.13435
40 Uribesalgo I, Hoffmann D, Zhang Y, Kavirayani A, Lazovic J, Berta J, Novatchkova M, Pai T, Wimmer RA, László V, Schramek D, Karim R, Tortola L, Deswal S, Haas L, Zuber J, Szúcs M, Kuba K, Dome B, Cao Y, Haubner BJ and Penninger JM: Apelin inhibition prevents resistance and metastasis associated with antiangiogenic therapy. EMBO Mol Med 11: e9266, 2019. PMID: 31267692. DOI: $10.15252 / \mathrm{emmm} .201809266$

41 Paluch EK and Raz E: The role and regulation of blebs in cell migration. Curr Opin Cell Biol 25: 582-590, 2013. PMID: 23786923. DOI: 10.1016/j.ceb.2013.05.005

42 Ikenouchi J and Aoki K: Membrane bleb: A seesaw game of two small GTPases. Small GTPases 8: 85-89, 2017. PMID: 27314434. DOI: $10.1080 / 21541248.2016 .1199266$

43 Madsen DH and Bugge TH: The source of matrix-degrading enzymes in human cancer: Problems of research reproducibility and possible solutions. J Cell Biol 209: 195-198, 2015. PMID: 25918222. DOI: $10.1083 /$ jcb.201501034

44 Bauvois B: New facets of matrix metalloproteinases MMP-2 and MMP-9 as cell surface transducers: Outside-in signaling and relationship to tumor progression. Biochim Biophys Acta - Rev Cancer 1825: 29-36, 2012. PMID: 22020293. DOI: 10.1016/j.bbcan.2011.10.001

45 Pahwa S, Stawikowski MJ and Fields GB: Monitoring and inhibiting MT1-MMP during cancer initiation and progression. Cancers (Basel) 6: 416-435, 2014. PMID: 24549119. DOI: 10.3390/cancers6010416

46 Guo Y, Pan W, Liu S, Shen Z, Xu Y and Hu L: ERK/MAPK signalling pathway and tumorigenesis (Review). Exp Ther Med 19: 1997-2007, 2020. PMID: 32104259. DOI: 10.3892/etm. 2020.8454

47 Jiang N, Dai Q, Su X, Fu J, Feng X and Peng J: Role of PI3K/AKT pathway in cancer: the framework of malignant behavior. Mol Biol Rep 47: 4587-4629, 2020. PMID: 32333246. DOI: $10.1007 / \mathrm{s} 11033-020-05435-1$

48 Kuba K, Sato T, Imai $\mathrm{Y}$ and Yamaguchi $\mathrm{T}$ : Apelin and Elabela/Toddler; double ligands for APJ/Apelin receptor in heart development, physiology, and pathology. Peptides 111: 62-70, 2019. PMID: 29684595. DOI: 10.1016/j.peptides.2018.04.011

Received September 14, 2020

Revised November 20, 2020

Accepted December 9, 2020 